\title{
Uma Revisão Sistemática do Uso da Gamificação no Ensino de Programação
}

\author{
Karine Heloise Felix de Sousa ${ }^{1}$, Lafayette B. Melo ${ }^{1}$ \\ ${ }^{1}$ Programa de Pós-Graduação em Tecnologia da Informação - Instituto Federal da \\ Paraíba (IFPB) João Pessoa - PB - Brasil \\ sousa.karinedacademico.ifpb.edu.br, la fayette.melo@ifpb.edu.br
}

\begin{abstract}
This article presents results of a systematic literature review (RSL) on the use of gamification in programming teaching in events and magazines in the country that use information technology in education. Primary studies from the years 2012 to 2020 were analyzed in order to reflect how this methodology is being applied and what are the mechanics/techniques used. A total of 23 studies were indexed, of which 7 were analyzed, to answer the research questions. Some conclusions were that the mechanics/techniques of gamification, such as competitions and cooperations, challenges, feedback, rewards were used in research and experiences in teaching programming in Computer Higher Education.
\end{abstract}

Resumo. Este artigo apresenta resultados de uma revisão sistemática da literatura (RSL) sobre o uso da gamificação no ensino de programação em eventos e revistas do país que utilizam informática na educação. Foram analisados estudos primários dos anos de 2012 a 2020 com o objetivo de refletir como esta metodologia está sendo aplicada e quais são as mecânicas/técnicas utilizadas. Um total de 23 estudos foram indexados, dos quais 7 foram analisados, para responder às questões de pesquisa. Algumas conclusões foram que as mecânicas/técnicas de gamificação, como as competições e cooperações, desafios, feedback, recompensas foram utilizados nas pesquisas e experiências no ensino de programação no ensino superior de Computação.

\section{Introdução}

O Currículo de Referência da Sociedade Brasileira de Computação aponta que a disciplina de programação é essencial para formação sólida dos discentes de diversos cursos de Computação [Sociedade Brasileira de Computação 1999].

A aprendizagem de programação não é fácil e, devido a isso, muitas universidades discutem com frequência seus currículos nos cursos de Ciência da Computação em busca de alternativas para diminuir o índice de evasão dos alunos. É comum observarmos pesquisas que apontam muitas evasões nestes cursos, fato que tem relação com as dificuldades de aprendizagem [Castro et al. 2003].

Aprender a programar envolve mais do que apenas entender a sintaxe de uma linguagem de programação e manipular estruturas de dados. Essa habilidade também requer a mobilização de um conjunto de conhecimentos prévios e de processos metacognitivos de modo a desenvolver soluções de problemas por meio de código [Prather et al. 2018].

No ensino de programação, o uso da gamificação como ferramenta motivacional e de transmissão de conhecimento tornou-se um assunto bastante difundido. Pesquisas 
mostram que a gamificação já foi utilizada no ensino de linguagem de programação e em conteúdos avançados [Kumar 2012].

Para o propósito desta pesquisa, foi realizada uma revisão sistemática da literatura (2012-2020) em relação ao uso da gamificação no ensino de programação, investigando uma diversidade de pesquisas publicadas sobre o assunto. Nessa perspectiva, o artigo apresenta questões para reflexão de como as mecânicas/técnicas da gamificação são abordados no ensino de programação. A partir dessas reflexões, identificaram-se tendências na utilização da gamificação que poderão servir como instrumento na prática do ensino de programação.

\section{Revisão da Literatura}

\subsection{Mecânicas/Técnicas de Gamificação}

Na gamificação, os elementos podem ser divididos conforme seu nível de abstração entre dinâmicas, mecânicas/técnicas e componentes [Werbach et al. 2012].

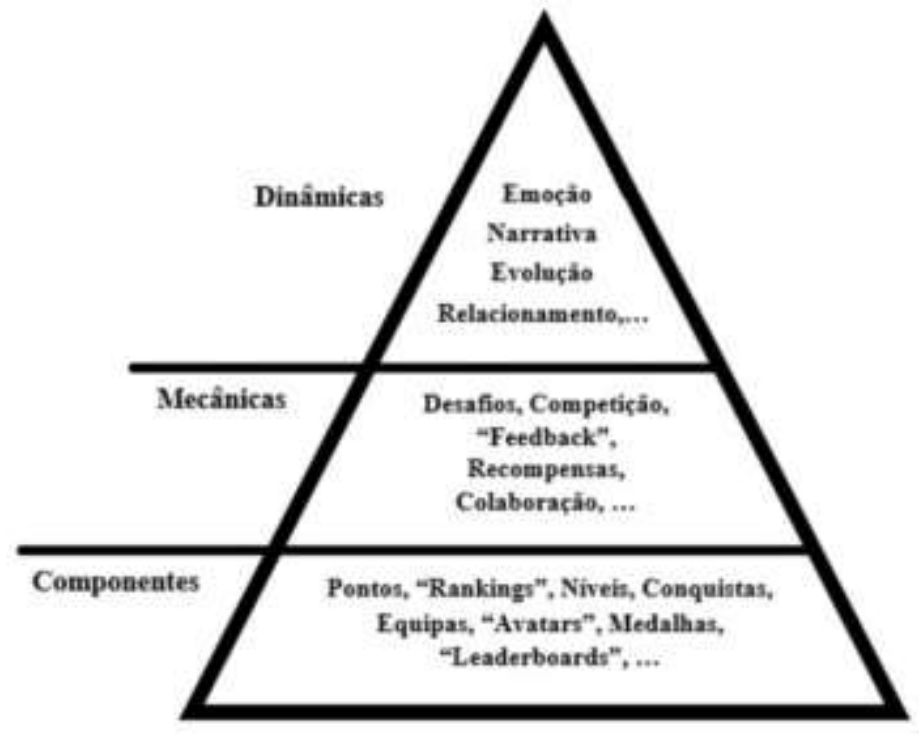

Figura 2 - Elementos da gamificação segundo Werbach (2012)

No segundo nível de abstração estão as técnicas/mecânicas (Figura 2), que são formas de induzir o jogador a realizar determinadas atividades dentro do sistema [Werbach et al. 2012]. Competições, cooperações, desafios, feedback, recompensas são exemplos de técnicas/mecânicas adotadas.

Competições e Cooperações são mecânicas utilizadas para promover a interação entre os jogadores [Werbach et al. 2012]. Na competição, os jogadores (ou grupos de jogadores) competem contra outros, estimulando a existência de um vencedor e de um perdedor. Já na cooperação, os jogadores trabalham em conjunto para atingir um objetivo compartilhado. Ambas podem ser utilizadas para estimular o relacionamento entre os usuários e despertar emoções.

Desafios são quebra-cabeças ou outras atividades que exigem esforço para serem resolvidas [Werbach et al. 2012]. Eles são importantes para guiar jogadores iniciantes ao mesmo tempo em que podem ser usados para adicionar profundidade e significado para os jogadores mais experientes [Zichermann et al. 2011]. Os desafios são comumente usados para fornecer um senso de progressão. 
Feedback retorna informações relevantes aos jogadores [Werbach et al. 2012]. Esse elemento é utilizado para gerar um ciclo de engajamento, em que o jogador é motivado a realizar uma determinada atividade e essa atividade fornece um feedback que reforça sua motivação para realizar novas atividades. Os principais usos do feedback envolvem o reforço das regras do sistema e o desdobramento das narrativas.

Recompensas são benefícios dados aos jogadores como forma de reconhecimento pelos seus esforços, como troféus que indiquem suas conquistas e itens que permitam a customização dos seus personagens. Além de demonstrar apreço pelo tempo que os jogadores investem, oferecer algo em troca reconhece seu sucesso e perspicácia. Recompensas são importantes porque criam medidas significativas do progresso, reforçam as regras do sistema e ajudam a manter o interesse do usuário ao longo do tempo [Ferrera 2012].

\subsection{Contextos de aplicação de mecânicas/técnicas de gamificação}

A gamificação corresponde ao uso de mecanismos de jogos, tais como: competição, desafio, interação social e recompensa, em contextos não relacionados a jogos (Kapp, 2012). Segundo Peixoto et al. (2015), mecânicas/técnicas de gamificação em softwares educacionais estão cada vez mais sendo utilizadas para apoiar a aprendizagem. Mas qual a vantagem de se usar gamificação na educação? Fardo (2013) argumenta que um dos benefícios é disponibilizar aos alunos um sistema no qual eles consigam visualizar o impacto de suas ações e sentir que estão aprendendo, no decorrer da disciplina. Assim, da mesma forma que acontece nos jogos, fica mais fácil entender que atingir pequenos objetivos contribui para alcançar um objetivo maior.

Assim, como argumenta Kaap (2012), a gamificação pode ser utilizada para promover a aprendizagem, e cada mecânicas/técnicas deve estar conectada entre si, pois, sozinhas, não são suficientes para transformar uma aula tradicional em uma experiência motivadora e engajadora, embora cada mecânicas/técnicas seja importante e fundamental no processo geral da gamificação.

Werbach e Hunter (2012) mostram que mecânicas/técnicas, estão os elementos que promovem a "ação", ou seja, é como se eles fossem a "engrenagem" do sistema que faz a gamificação funcionar no decorrer da execução. Dentre eles, podemos citar: desafios, sorte, cooperação e competição, feedback, aquisição de recursos, recompensas, transações, turnos e estados de vitória.

$\mathrm{Na}$ área de educação, também é possível aplicar mecânicas/técnicas de gamificação, como fez o CodeSchool, que é uma plataforma de aprendizado on-line que ensina diversas linguagens de programação e design para web através de vídeos e exercícios interativos [CodeSchool 2021]. Apenas alguns cursos oferecidos pela plataforma são gratuitos, nos quais há desafios que, ao serem completados, dão recompensas aos alunos, que podem ser utilizadas para "comprar" respostas quando o aluno possui dificuldade em concluir um desafio - o aluno ganha recompensas ao completar os níveis [Klock et al. 2014].

O URI Online Judge é um projeto gratuito realizado pela Universidade Regional Integrada para promover a programação de computadores na prática e auxiliar no compartilhamento do conhecimento [URI Online Judge 2021]. Esse projeto propõe problemas e permite que os estudantes resolvam tais problemas com algoritmos nas linguagens C++ ou Java. No URI Online Judge existem mecânicas/técnicas de 
gamificação: recompensas, os desafios e a competição de acordo com a quantidade de problemas resolvidos pelos alunos [Klock et al. 2014].

\subsection{Trabalho correlacionados}

Como trabalhos correlatos é possível citar as seguintes pesquisas.

Aureliano et al. (2012) desenvolveram e publicaram um estudo sobre o ensinoaprendizagem da lógica de programação para iniciantes cujo objetivo foi fornecer uma análise abrangente e sistemática dos trabalhos científicos publicados no Simpósio Brasileiro de Informática na Educação (SBIE) e no Workshop de Informática na Escola (WIE). Para isso, utilizaram uma metodologia de RSL visando extrair, catalogar, analisar e sintetizar dados de todos os artigos publicados em 10 edições (2002-2011) desses eventos.

Medeiros et al. (2013), semelhantemente, apresentou uma RSL para o ensino e aprendizagem da lógica de programação com jogos digitais no contexto nacional. Este estudo desenvolveu um processo similar ao apresentado por Medeiros, Silva e Aranha (2013), aplicando a técnica de pesquisa secundária para realizar uma análise abrangente e sistemática da pesquisa em jogos digitais para o ensino e aprendizagem da lógica de programação no cenário internacional.

Outro estudo que fez uso da RSL foi a pesquisa de Silva et al. (2014). No trabalho produzido, analisaram 29 estudos primários com o objetivo de investigar a eficácia dos jogos digitais para o ensino e aprendizagem da lógica de programação.

Por outro lado, Vahldick et al. (2015) apresentam uma revisão sistemática dos jogos usados para melhorar as competências dos alunos em tarefas de introdução à programação. Para realizar a revisão sistemática, o trabalho pesquisou em bases de dados comuns e também em banco de dados comerciais, tais como AppStore, GooglePlay e sites que disponibilizam tarefas de programação, por exemplo escrito em Flash. As questões de pesquisa elencadas pelo trabalho foram 1) quantos jogos estão disponíveis para auxiliar no entendimento de programação introdutória depois dos anos 2000? 2) quais são as habilidades e tópicos cobertos por esses jogos e 3) quais características estão faltando para um melhor aprendizado de programação introdutória?

Diferentemente, Agbo et al. (2019) apresentam uma revisão sistemática com o objetivo de identificar como o pensamento computacional tem sido usado no ensino de introdução à programação. Especificamente, o artigo busca identificar os artigos que discutiram a abordagem pensamento computacional. Além disso, objetiva classificar as diferentes abordagens e ferramentas usadas para ensinar programação.

Por outro lado, Shahid et al. (2019) apresentam uma revisão da literatura sobre o desenvolvimento de jogos relacionados à programação. Dentre as características relacionados ao desenvolvimento de jogos abordados, pode-se destacar (1) caracterização dos tópicos de programação tipicamente utilizados em projeto de jogos para a educação (2) linguagens de programação utilizadas e (3) características com respeito ao framework MDA (Mechanics, Dynamic e Asthetic). Os mecanismos dizem respeito a um componente particular do jogo que representam dados e algoritmos. Por sua vez, a dinâmica se refere ao ciclo de vida dos mecanismos que funciona para engajar o usuário ao fornecer entradas e receber feedback continuamente. Por fim, a Estética refere-se a emoção do usuário ao utilizar o jogo 


\section{Método de Pesquisa}

Adotou-se a Revisão Sistemática da Literatura (RSL) como metodologia para esta pesquisa. De acordo com Sampaio e Mancini (2017), algumas das vantagens de uma revisão sistemática são a possibilidade de ser replicável e de identificar lacunas na pesquisa atual. Para a realização desta pesquisa, foram executadas três fases distintas: Elaboração, Estruturação e Resultados, com suas respectivas etapas apresentadas na Figura 1.

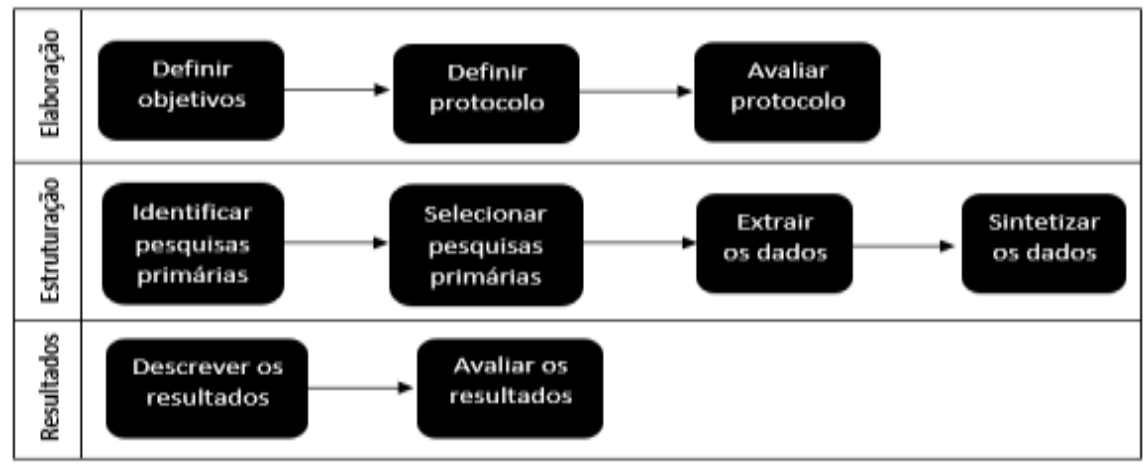

Figura 1 - Fases da Revisão Sistemática

$\mathrm{Na}$ Elaboração, foram definidos o objetivo e as questões de pesquisa, além da definição do protocolo (https://drive.google.com/file/d/1K-NHTzp2aJR8MV8yIYErmrIyetT-o9k/view?usp=sharing ). Na fase de Estruturação, procedeu-se à seleção dos estudos e à análise das questões de pesquisa, bem como à sintetização dos dados coletados. Definiu-se a string de busca nos engenhos de busca de artigos científicos mais significativos da área, selecionando-os a partir dos critérios de inclusão. Na última fase, resultados foram tabulados e foram classificadas as incidências das questões e como estão relacionadas com cada um dos artigos selecionados.

\subsection{Objetivo e Questões de Pesquisa}

O objetivo central deste trabalho foi o de compreender como a gamificação vem sendo aplicada no ensino de programação. Para tanto, para alcançar esse objetivo, foram elaboradas as questões de pesquisa:

(Q1) Quais as mecânicas/técnicas de gamificação são utilizadas no aprendizado de programação?

(Q2) Como as mecânicas/técnicas de gamificação são utilizadas no aprendizado de programação?

\subsection{Estratégia de busca}

Na construção da string de busca, foram identificados os constructos que deveriam estar contidos nos trabalhos pesquisados, em seguida foram identificados os sinônimos e ou termos alternativos dessas palavras-chaves. A tabela 1 apresenta a string gerada.

\section{Tabela 1. String de busca}

("gamificação” AND “ensino de programação”) OR ("gamification” AND "programming teaching") 


\subsection{Coleta dos Trabalhos e Fontes de Busca}

A coleta dos trabalhos ocorreu em buscas nas fontes de dados digitais que reúnem e publicam trabalhos de excelência realizados por profissionais e pesquisadores na área de Informática na Educação e procuram disseminar ferramentas, métodos e práticas que auxiliem no uso efetivo da tecnologia no processo de ensino e aprendizagem de programação entre os anos de 2012 a 2020, pois têm uma periodicidade maior, com melhor atualização.

As fontes de busca utilizadas para realização deste trabalho foram escolhidas pelo grau de relevância científica considerando a área de concentração da pesquisa e pela acessibilidade. É importante destacar que os motores de busca presentes nestas fontes apresentam opções de parametrização que permitiram que o filtro dos trabalhos pesquisados fosse utilizado na seleção dos trabalhos de acordo com os critérios de inclusão.

Foi utilizada a string de busca na língua portuguesa de modo a conduzir as pesquisas na Revista Brasileira de Informática na Educação (RBIE) e no Simpósio Brasileiro de Informática na Educação (SBIE).

As bibliotecas digitais utilizadas na busca automatizada, com a quantidade de artigos indexados, foram: ACM Digital Library, 10; IEEEXplore Digital Library, 08; SBIE - Simpósio Brasileiro de Informática na Educação, 02 e RBIE - Revista Brasileira de Informática na Educação com 03 artigos retornados.

\subsection{Critérios de Inclusão}

Para a inclusão de um trabalho na pesquisa, foi determinada sua relevância em relação às questões de pesquisa, considerando os critérios de inclusão, a saber: (i) ter sido publicado no ACM, IEEExplore, SBIE e RBIE; (ii) ter sido publicada entre os anos de 2012 a 2020; (iii) artigos cuja língua seja inglês, espanhol e português; (iv) artigos que sejam short papers ou full papers; (v) artigos que sejam de pesquisas primárias (excluídos surveys, mapeamentos e revisões sistemáticas).

Após a aplicação dos critérios de inclusão pela leitura sistematizada dos títulos, palavras-chave, resumos e eventualmente do corpo do trabalho, dos 23 trabalhos retornados na busca realizada, 7 deles foram incluídos para a etapa de extração dos dados. Para que o estudo fosse incluído, foi necessária uma análise minuciosa, considerando os critérios de inclusão. A tabela 2 apresenta a quantidade de trabalhos incluídos por engenho de busca. Todos os trabalhos incluídos podem ser encontrados na planilha: https://docs.google.com/spreadsheets/d/1bQkTAcXUAwu2MA76TUnWczLZS8MyO_1 CaGDh8IgH2-c/edit?usp=sharing .

Tabela 2. Trabalhos selecionados, Incluídos e Excluídos.

\begin{tabular}{|l|c|c|c|}
\hline Fontes de busca & Selecionados & Incluídos & Excluídos \\
\hline IEEEXplore & 08 & 03 & 05 \\
\hline ACM Digital & 10 & 03 & 07 \\
\hline SBIE & 02 & 00 & 02 \\
\hline RBIE & 03 & 01 & 02 \\
\hline
\end{tabular}

\subsection{Extração e Sintetização dos Dados}

Após a seleção dos estudos incluídos, os artigos foram submetidos à fase de extração e sintetização dos dados, na qual foram lidos por completo e extraídos os seus conteúdos e 
incluídos na planilha: título do artigo, autores, breve resumo do trabalho, ano da publicação, idioma, técnica da gamificação e utilização da gamificação. Uma vez definidos todos os dados, os autores iniciaram a extração das informações dos trabalhos selecionados na revisão sistemática.

\section{Análise das questões de pesquisa}

Esta seção mostra a revisão sistemática das evidências encontradas nos estudos primários como respostas aos questionamentos levantados nas duas questões propostas.

\section{Q1 - Quais as mecânicas/técnicas de gamificação são utilizadas no aprendizado de programação?}

Ao analisar a Questão 1, as mecânicas/técnicas de gamificação exploradas nos estudos analisados foram: competições e cooperações, desafios, feedback, recompensas.

No experimento de Carreño-León et al. (2018), ofereceram-se recompensas pela realização de tarefas, aproveitando a competitividade, tornando visível as recompensas entre os jogadores e tornando as tarefas existentes mais atraentes que antes geralmente poderiam ser entediantes.

As mecânicas/técnicas gamificadas usados pelo UDPiler no estudo de Marín et al. (2018) foram as medalhas como meio de recompensa por cada desafio alcançado no desenvolvimento das atividades.

No estudo de Jamet et al. (2018), integrar os conceitos de gamificação na sala de aula, estes métricas tornarão possível coletar informações relevantes sobre alunos, dandolhes feedback oportuno sobre seus desempenhos, bem como fornecer dados analíticos úteis para partes interessadas em seu desempenho durante o desenvolvimento do assunto.

Padirayon (2019) em seu sistema de aprendizagem gamificado, fez com que os alunos estudassem os tópicos e respondessem aos questionários e exercícios com pontuação, assim recebendo uma recompensa em forma de emblema reconhecendo seu nível no assunto.

Figueiredo et al. (2020) em seu sistema de tutoria inteligente utilizou mecânicas/técnicas de gamificação do feedback imediato como forma de orientar o aluno em seu aprendizado em programação, assim reduzindo falta de motivação dos alunos.

No conjunto de atividades descrito no trabalho de Figueiredo et al. (20 20) utilizando algumas mecânicas/técnicas de gamificação, como a caixa surpresa que seria um desafio e o torneio de eliminação que usa competição, recompensas e feedbacks.

Ribeiro et. al. (2020), explica que juiz online CodeBench foi gamificado utilizando-se mecânica/ténicas de gamificação como desafio e recompesas, nas quais definiram as metas do jogo, suas regras e a forma de interação do sistema.

\section{Q2 - Como as mecânicas/técnicas de gamificação são utilizadas no aprendizado de programação?}

A técnica de gamificação implementada em sala de aula por Carreño-León et al. (2018), em curso introdutório de programação, foi usada como suporte para aumentar a capacidade na resolução de problemas.

O estudo de Marín et al. (2018) foi feito utilizando uma plataforma gamificada denominada UDPiler, que permite enfrentar diferentes desafios de programação, com 
quatro tipos de desafios: múltipla escolha, completando ou corrigindo um trecho de código, codificação para produzir uma saída esperada e ganhar um minijogo por meio de codificação. Ainda neste estudo, cada aluno que obtém uma resposta correta recebe uma medalha e obtém alguns pontos, que são somados a uma classificação na programação curso. As medalhas são imagens com conteúdo relacionado, como a imagem de um superherói da Marvel.

Pode-se destacar o estudo de Jamet et al. (2018), que se baseia na metodologia 6 D's que é uma ferramenta para a elaboração de programas de treinamento com a geração de resultados mensuráveis. Foram utilizadas nessa metodologia as mecânicas/técnicas do relacionamento e competição da gamificação no aprendizado de programação do primeiro ano curso de computação.

Com isso, Padirayon (2019) implementa as mecânicas/técnicas de gamificação no aprendizado em programação em: exercícios, questionários, tarefas, respostas e apresentação de vídeo e exames. Algumas gamificações ocorrem em objetos como imagens, áudios, vídeos, animações e multimídia e são usadas principalmente para criar experiências sensoriais.

No experimento de Figueiredo et al. (2020) foi utilizado um sistema de tutoria inteligente com as mecânicas/técnicas de gamificação, a fim de construir uma estratégia de ensino para ajudar os alunos, reduzindo o alto índice de fracasso e a falta de motivação nos cursos introdutórios programação. O uso de gamificação em sala de aula mostrou um boa aceitação e interesse dos alunos.

Ribeiro et al. (2020) usou uma versão de juiz online com mecânicas/técnicas gamificadas que foram aplicadas em 11 turmas ofertadas nos cursos da área de ciências exatas e engenharias na Universidade Federal do Amazonas. Durante o primeiro semestre letivo de 2017/1, os dados foram comparados com alunos das turmas ofertadas em 2016/1 dos mesmos cursos, mas com o apoio da versão original do juiz online, sem gamificação. As turmas 2016/1 e 2017/1 eram compostas por estudantes com desempenhos semelhantes, e foi possível ver uma mudança no desempenho com a utilização da ferramenta da gamificação do juiz online, o qual contribuiu no desempenho na disciplina Introdução à Programação.

Para aumentar a motivação dos alunos Figueiredo et al. (200) utiliza um jogo que possui uma caixa surpresa que é um conjunto de atividades do qual um é desenhado para o aluno resolver. As atividades eram avaliadas com pontos de acordo com o grau de dificuldade. Os pontos serão atribuídos ao aluno após a resolução correta. Em um torneio de eliminação, onde dois alunos competiam em resolver um exercício. $\mathrm{O}$ aluno que perdia era eliminado do torneio. $\mathrm{O}$ aluno vencedor seguia para o próximo estágio. Os pontos eram atribuídos para cada fase alcançada em o torneio.

\section{Conclusões}

A gamificação tornou-se uma estratégia interessante para o processo de aprendizado de programação, pois consegue atrair a atenção e manter os alunos ativos em sala de aula, ajudando a resolver desafios de maneira lúdica e, principalmente, trabalhando de modo colaborativo para a construção do conhecimento. Nesta revisão sistemática foi possível observar a metodologia presente nos trabalhos e a forma como produzem resultados promissores quando bem planejados. 
Ao todo, foram selecionados 23 trabalhos, os quais foram submetidos aos processos de seleção preliminar, seleção final e extração de resultados. Após a análise, constatou-se que 07 das publicações que abordam gamificação apresentam resultados em seus experimentos de aprendizado em programação.

Observa-se que, os benefícios descritos nos resultados dos estudos que examinaram a aplicação da gamificação na programação, como no relato de Zanchett et al. (2015), descrevem que os objetivos educacionais podem ser atingidos enquanto os estudantes se divertem.

Os experimentos se alinham com o trabalho de Penteado et al. (2014), uma vez que se pode concluir que de fato a gamificação traz benefícios e contribuições na programação. Um dos benefícios observados que se pode citar é o entusiasmo dos discentes ao fazer uso de um recurso metodológico e lúdico que está fortemente relacionado ao contexto extraclasse do qual muitos participam.

O objetivo maior deste estudo foi oferecer uma visão acerca dos trabalhos realizados pelos pesquisadores nos anos de 2012 a 2020 sobre a temática da gamificação, com especial destaque para as pesquisas no ensino de programação. Nesse nível, observamos que as experiências são ricas e os resultados, na maioria dos estudos, foram promissores, permitindo um maior engajamento dos sujeitos, aumento no aprendizado, maior motivação e aprimoramento de habilidades.

Para investigações futuras, colocamos a possibilidade de ampliar a base de dados de trabalho através de um comparativo entre artigos desenvolvidos no país e fora dele. Dessa forma, será possível verificar se os resultados são os mesmos, ou se os investigadores utilizam as mesmas metodologias e tecnologias em suas atividades de gamificação no ensino de programação.

\section{Referências}

Agbo, F. J., Oyelere, S. S., Suhonen, J., and Adewumi, S. (2019). A systematic review of computational thinking approach for programming education in higher education institutions. In Proceedings of the 19th Koli Calling International Conference on Computing Education Research. Koli Calling'19, New York, NY, USA. Association for Computing Machinery.

Aureliano, V. C. O.; Tedesco, P. C. A. R. Ensino-aprendizagem de Programação para Iniciantes: uma Revisão Sistemática da Literatura focada no SBIE e WIE. In: Simpósio Brasileiro de Informática na Educação, 2012, Curitiba, Anais... Curitiba. 2012.

Carreño-León, M.; Sandoval-Bringas, A.; Álvarez-Rodríguez, F.; Camacho-González Y. Gamification technique for teaching programming, 2018 IEEE Global Engineering Education Conference (EDUCON), Tenerife, 2018, pp. 2009-2014, doi: 10.1109/EDUCON.2018.8363482.

Castro, C. T., Castro Junior, A., Meneses, C., B., M. e Rauber, M. (2003) Utilizando Programação Funcional em Disciplinas Introdutórias de Computação, In: XI Workshop de Educação em Computação - WEI, Campinas/SP.

Codeschool. Site Oficial. Disponível em: <https://www.codeschool.com>. Acesso em: 26 jun. 2021. 
Fardo, M. L.. A gamificação como estratégia pedagógica: estudos de elementos dos "games" aplicados em processos de ensino e aprendizagem. Dissertação de mestrado em educação, Universidade de Caxias do Sul, Caxias do Sul, RS, 2013.

Ferrera, J. Playful Design: Creating game experiences in everyday interfaces. New York: Rosenfeld Media, LLC, 2012.

Figueiredo, José; García-Peñalvo, Francisco José. 2020. Intelligent Tutoring Systems approach to Introductory Programming Courses. In Eighth International Conference on Technological Ecosystems for Enhancing Multiculturality (TEEM'20). Association for Computing Machinery, New York, NY, USA, 34-39. DOI:https://doi.org/10.1145/3434780.3436614.

Figueiredo, José; García-Peñalvo, Francisco José, "Increasing student motivation in computer programming with gamification," 2020 IEEE Global Engineering Education Conference (EDUCON), 2020, pp. 997-1000, doi: 10.1109/EDUCON45650.2020.9125283.

Figueiredo, K. da S. Proposta de gamificação de disciplinas em um curso de sistemas de informação. 2015.

Jamet E.; Valencia, K.; Figueroa, I. Objectives and Behaviors for a Gamification Strategy for First Year Programming Courses, 2018 37th International Conference of the Chilean Computer Science Society (SCCC), Santiago, Chile, 2018, pp. 1-6, doi: 10.1109/SCCC.2018.8705162.

Kapp, K. (2012). The gamification of learning and instruction: game-based methods and strategies for training and education. John Wiley \& Sons.

Klock, Ana Carolina Tomé; Carvalho, Mayco Farias de; Rosa, Brayan Eduardo, GASPARINI, Isabela. Análise das técnicas de Gamificação em Ambientes Virtuais de Aprendizagem. Disponível em <https://seer.ufrgs.br/renote/article/view/53496/0>. Acesso em 26 de jun. 2021.

Kumar, B. and Khurana, P. Gamification in education-learn computer programming with fun, International Journal of Computers and Distributed Systems, v. 2, n. 1, p. 46-53, 2012.

Marín, B.; Frez, J.; Cruz-Lemus, J.; Genero, M. 2018. An Empirical Investigation on the Benefits of Gamification in Programming Courses. <i>ACM Trans. Comput. Educ. $</ \mathrm{i}>\quad 19, \quad 1, \quad$ Article 4 (January 2019), 22 pages. DOI:https://doi.org/10.1145/3231709.

Medeiros, T. J.; Silva, T. R.; Aranha, E. H. S. "Ensino de programação utilizando jogos digitais: uma revisão sistemática da literatura". In: Revista Novas Tecnologias na Educação - RENOTE, v.11, n.3. Anais... Porto Alegre. 2013.

Padirayon, Lourdes M.. 2019. The Designed Gamification Application Architecture and Elements for a C\# Programming Course. In <i>Proceedings of the 2019 4th International Conference on Multimedia Systems and Signal Processing $</ \mathrm{i}>$ $(<\mathrm{i}>$ ICMSSP 2019</i $>$ ). Association for Computing Machinery, New York, NY, USA, 67-72. DOI:https://doi.org/10.1145/3330393.3330414.

Peixoto, M. M., Silva, C., Gonçalves, E., \& Vilena, J.. Um Mapeamento Sistemático de Gamificação em Software Educativo no Contexto da Comunidade Brasileira de 
Informática na Educação. In Anais do XXI Workshop de Informática na Escola. 2015. doi:10.5753/cbie.wie.2015.584

Penteado, C.; Damasceno, E. Contribuições de jogos digitais no ensino da lógica de programação para o ensino médio integrado em informática. Revista E.T.C. Logomarca. Edição 01. 2014.

Prather, J., Pettit, R., McMurry, K., Peters, A., Homer, J., \& Cohen, M. (2018). Metacognitive Difficulties Faced by Novice Programmers in Automated Assessment Tools. In Proceedings Ribeiro et al. RBIE 28 - 2020490 of the 2018 ACM Conference on International Computing Education Research, 41-50. doi:10.1145/3230977.3230981.

Ribeiro, Ralph Breno Silva et al. Investigação Empírica sobre os Efeitos da Gamificação de um Juiz Online em uma Disciplina de Introdução à Programação. Revista Brasileira de Informática na Educação, [S.1.], v. 28, p. 461-490, jun. 2020. ISSN 2317-6121. Disponível em: <https://www.br-ie.org/pub/index.php/rbie/article/view/v28p461>. Acesso em: 24 jan. 2021. doi:http://dx.doi.org/10.5753/rbie.2020.28.0.461.

Sampaio, R. F., Mancini, M. C. (2007). Estudos de revisão sistemática: um guia para síntese criteriosa da evidência científica. Brazilian Journal of Physical Therapy, 11, 83-89

SBC (1999) - Sociedade Brasileira de Computação. Currículo de Referência da SBC para Cursos de Graduação em Computação e informática.

Shahid, M., Wajid, A., Haq, K., Saleem, I., and Shujja, A. (2019). A review of gamification for learning programming fundamental, cited By 0 .

Silva, T.; Medeiros, T.; Aranha, E. Jogos Digitais e Aprendizagem de Programação: uma revisão sistemática da literatura. In.: Simpósio Brasileiro de Informática na Educação, p. 692-701, 2014, Brasília. Anais... Brasília, 2014.

Uri Online Judge. Disponível em: <https://www.urionlinejudge.com.br>. Acesso em: 26 jun. 2021.

Vahldick, A., Mendes, A., and Marcelino, M. (2015). A review of games designed to improve introductory computer programming competencies, volume 2015-February, cited By 15.

Werbach, K., \& Hunter, D. (2012). For the win: How game thinking can revolutionize your business. Wharton Digital Press.

Werbach, K. Hunter, D. (2016). "For the Win: How Game Thinking Can Revolutionize Your Business", Wharton Digital Press, Philadelphia.

Zanchett, G.; Vahldick, A.; Raabe, A. Jogos de Programar como uma Abordagem para os Primeiros Contatos dos Estudantes com a Programação. In.: Workshop do Congresso Brasileiro de Informática na Educação, 2015, Recife. Anais... Recife, 2015.

Zichermann, G.; Cunningham, C. Gamification by Design: Implementing game mechanics in web and mobile apps. Sebastopol: O’Reilly Media, Inc., 2011. 